\title{
Effect of Global Postural Reeducation Exercise on Pain and Hip Muscle Flexibility in Patients with Chronic Low Back Pain and Movement Control Dysfunction
}

\author{
Bahram Sheikhi ${ }^{*}$ \\ 'Department of Biomechanics and Sports Injuries, Faculty of Physical Education and Sports Sciences, Kharazmi University, \\ Tehran, Iran
}

\author{
*Correspondence to \\ Bahram Sheikhi, \\ Department of Biomechanics and \\ Sports Injuries, Faculty of Physical \\ Education and Sports Sciences, \\ Kharazmi University, Tehran, Iran \\ Tel: +989376569397 \\ +982122228001 \\ Email: sheikhibahram@gmail.com
}

Received November 18, 2018 Accepted September 25, 2019 Published online December 31, 2019

\begin{abstract}
Introduction: Patients with non-specific low back pain (NSLBP) and movement control dysfunction demonstrate alternation in hip muscles flexibility and spinal movement patterns. Therapeutic modalities that augment hip muscles flexibility could help these patients. The aim of this study was to investigate the effect of global postural reeducation (GPR) on pain and hip muscle flexibility in patients with NSLBP and movement control dysfunction.

Materials and Methods: A total of 27 men with a mean age of $31.21 \pm 2.5147$ years, height of $166.44 \pm 6.11 \mathrm{~cm}$, and weight of $64.21 \pm 5.25 \mathrm{~kg}$ participated in this study. The visual analogue scale (VAS) was used to evaluate pain. The flexibility of hip muscles (rectus femoris, tensor fasciae latae, external rotators and hamstring) was measured using universal goniometer. All data were assessed at baseline and after the intervention. The Shapiro-Wilk test and paired t test were used for statistical analysis at significance level of $P=0.05$.

Results: Our results revealed a decline in pain $(P<0.004)$ and an increase in the flexibility of the hamstring muscles in the right $(P<0.003)$ and left $(P<0.003)$ legs. There were no statistically significant differences in the flexibility of rectus femoris muscle, external rotators, and tensor fasciae latae.

Conclusion: The results suggest that GPR had a significant effect on the level of pain. Further, it affected the flexibility of hamstring muscles in legs. Using GPR is recommended for pain relief and improving the flexibility of hamstring muscles in patients with NSLBP.

Keywords: Chronic non-specific low back pain, Motor control, Global postural reeducation, Flexibility
\end{abstract}

Please cite this article as follows: Sheikhi B. Effect of Global Postural Reeducation Exercise on Pain and Hip Muscle Flexibility in Patients with Chronic Low Back Pain and Movement Control Dysfunction. Int J Basic Sci Med. 2019;4(4):148154. doi: $10.34172 /$ ijbms.2019.05.

\section{Introduction}

Low back pain (LBP) is a common multifactor musculoskeletal disorder in today's societies, ${ }^{1,2}$ which is experienced by over 70 to $80 \%$ of people at least once in their life. ${ }^{3}$ Non-specific low back pain (NSLBP) refers to muscle tension or local stiffness in the region below the ribs and above the gluteal sulcus, which can be defined with or without leg pain. ${ }^{4}$ Although most of the time, NSLBP improves spontaneously, half of the patients with this disease have a long history of back pain or several episodes of back pain. According to the current reports,
\end{abstract}

this group of patients alone account for $80 \%$ of the costs associated with back pain treatment. ${ }^{5}$

According to Luomajoki et al, one possible underlying mechanism of NSLBP is movement control impairment (MVCI) ${ }^{6}$ which can be due to pain, abnormal tissue loading, lack of proprioceptive awareness and, possibly, the lack of a withdrawal reflex motor response. ${ }^{6}$ The consensus is increased muscle activity in these individuals as a stabilization mechanism. ${ }^{7,8}$

Research has shown that around $70 \%$ of patients with nonspecific back pain suffer

(c) 2019 The Author(s); Published by Zabol University of Medical Sciences. This is an open-access article distributed under the terms of the Creative Commons Attribution License (http://creativecommons.org/licenses/by/4.0), which permits unrestricted use, distribution, and reproduction in any medium, provided the original work is properly cited. 
from various mechanical disorders including changes in the strength and length of the spinal muscles, diminished strength of spinal muscles, asymmetry of internal and external rotation of the hip, and pes planus.. In previous studies on these patients, the relationship between these mechanical disorders and intensity of NSLBP has been measured. ${ }^{4}$ In a study conducted to investigate the relationship between mechanical factors and intensity of back pain, the strength and stamina of the back muscles and those surrounding the hip had a close relationship with the intensity of back pain. ${ }^{4}$

Furthermore, NSLBP causes the patient to experience a vicious cycle, where the patients with chronic back pain (more than three months), experience movement constriction, whereby their physical activity becomes severely restricted, further increasing muscle weakness. ${ }^{10}$ Although the effect of interventions on movement defects in patients with NSLBP has not been evaluated systematically so far, most studies have reported a relationship between pain and mobility constriction as well as altered movements and postural patterns with the intensity of back pain. In a study by Stevenson et al on factory workers, it was found that the flexibility of body muscles generally affects the incidence of NSLBP. ${ }^{11}$

Exercise can play a significant role in treating NSLBP. The effectiveness of exercise therapy has been reported as a non-invasive and non-pharmacological method for treating NSLBP across various studies. ${ }^{12,13}$

Studies have suggested that exercise significantly reduces the relapse of NSLBP. ${ }^{11}$ In addition, researchers have mentioned various therapeutic exercises for each individual factor (flexibility as well as strength and stamina of muscles), where each of them is specific only to one factor. ${ }^{12,13}$ If the therapist applies the special therapeutic exercises to the patient to improve each factor, it necessitates a long time for treatment. Further, the patient does not have the ability to do all the exercises simultaneously. For being completely recovered, most of the factors mentioned should be improved among patients with NSLBP; therefore, a kind of exercise is required which is able to treat most disordered muscles simultaneously.

In the research by van der Velde and Mierau, it was found that a 6-week exercise program improved the flexibility of muscles in patients with chronic nonspecific NSLBP. ${ }^{12}$ Gordon and Bloxham systematically investigated the effects of exercise and physical activity on NSLBP. They observed that these exercises can improve the flexibility and range of motion of muscles. ${ }^{13}$ The results of a study by Leo Rathinaraj et al on the effect of segmental stability of the spine with an existing program in patients with mechanical LBP indicated that exercise plays a vital role in mitigating NSLBP. ${ }^{14}$ Nezhad Roomezi et al studied the effect of core stability exercises on the pain and performance of female patients with NSLBP and observed a significant improvement in the extent of pain and performance of the women with NSLBP. ${ }^{15}$

One of the therapeutic methods that have recently attracted attention is global postural reeducation (GPR) method which was developed in France by Souchard et al. ${ }^{16}$ This therapeutic method has been developed based on an integrated idea of the system of muscular chains, which can face shortening resulting from situational, behavioral, and psychological factors. ${ }^{17}$ This method has been developed based on identifying posterior and anterior muscular chain and the general stretching of antigravity muscles. ${ }^{17}$

Although this method is widely used across different countries, only a few studies support its clinical and theoretical effects. The studies that have been conducted with regards to GPR training indicated that this method might be effective in treating some musculoskeletal diseases and disorders including NSLBP, disc herniation, and ankylosing spondylitis. ${ }^{18}$ The results indicated that GPR intervention in people with back pain resulted in further improvement of pain and disability compared with stability training. ${ }^{17}$ Some studies have been developed on functional exercises over the past recent years, the majority of which have mainly dealt with investigating balance disorders and have less addressed disorders of motion and movement control. ${ }^{19,20}$

Therefore, based on what has been mentioned and suggestions of previous researches, the present study aims to investigate the effect of a six-week period of GPR exercises on the pain and flexibility of some hip muscles of men with NSLBP suffering from lumbar MVCI.

\section{Materials and Methods}

The present study was a quasi-experimental study with pre- and post-intervention design.

\section{Inclusion and Exclusion Criteria}

Inclusion criteria were the diagnosis of NSLBP and lumbar MVCI, ${ }^{21}$ being 30-35 years of age, being classified as medium-risk subgroup (patients with an unfavorable prognosis with high levels of physical prognostic indicators appropriate for physiotherapy were excluded) using the STarT Back Screening Tool, ${ }^{22}$, having no history of macro trauma and acute lesions, having no history of surgery in the back region and lower extremities, not having functional or congenital kyphosis (using kyphometer) ${ }^{23}$ and scoliosis (using scoliometer), ${ }^{24}$ having no history of receiving physical therapy over the past three months, and not receiving pharmacotherapy. The exclusion criteria included unwillingness of the patient to continue the therapeutic program, not attending the therapeutic sessions, and having diseases that disrupted participation in the study.

Sample Population and Study Design

Using G-Power software, 27 men with NSLBP ${ }^{25,26}$ and deficit in performing Luomajoki and colleagues' 
movement control tests ${ }^{27}$ were chosen for this research. In this study, purposive sampling method was used and after baseline testing, the patients were allocated to the experimental groups.

After recording the demographic characteristics of the patients, those who had the initial inclusion criteria with NSLBP (Quebec questionnaire) ${ }^{28}$ and movement control deficit were included in the research. All of the applicants were tested in terms of movement control so that the statistical population of the research would consist of patients with NSLBP suffering from movement control deficit. ${ }^{21,27}$ In the pre-and post-test stages, the evaluation of flexibility and pain was performed on all the subjects under the same conditions using the following method. The pain intensity was measured using visual analog scale (VAS), while the flexibility of rectus femoris, hamstring, external rotators, and tensor fasciae latae muscles was measured by a goniometer, which was performed by the researcher before and after the intervention. After pretest evaluation, the experimental group performed GPR exercises for six weeks. Two days after the completion of the exercises, post-test was done under pretest conditions, and the data were analyzed further.

\section{Quebec Questionnaire}

At the beginning of the study, the Québec Back Pain Disability Scale (QBPDS) which included 20 items, with each having a predetermined score, was used for sampling. For this purpose, the subjects filled this questionnaire. Then, they were examined by specialists for clinical assessment. Those who did not meet the conditions of running the training protocol and intended tests, according to the specialists, and had a QBPDS score of lower than 15 were excluded from the research. ${ }^{28}$

\section{Movement Control Test}

In this research, movement control tests were performed after verification by the specialists and filling the questionnaire by the subjects. The subjects performed movement control tests which included six tests (Table 1). When implementing these tests, if the subject had an incorrect performance, the proper method was explained, and if he learned the proper movement but was still unable to do it, the person was considered to have a movement control deficit. In addition, those who had at least two deficits in these tests were included in the present study. ${ }^{29}$

\section{Assessing the Intensity of Back Pain}

The intensity of back pain was measured by VAS. This scale has been plotted and graded as a $10-\mathrm{cm}$ line, which was used for measuring the intensity of perceived pain in this study. In this scale, zero represents the absence of pain, 1-3 shows mild pain, 4-6 denotes moderate pain, and 7-10 indicates severe pain. In this study, the patients specified their perceived pain (which they sensed most of the time) by indicating a position on the continuum as the method of measuring pain. ${ }^{31}$

\section{Evaluating Muscle Flexibility}

Flexibility of hip muscles was measured using a universal goniometer (Patterson Medical) (Table 2).

\section{The GPR Protocol}

In the present study, for the GPR method, positions which involved mainly the posterior chain length were chosen as this chain is shortened in patients with NSLBP. This method involves lying, sitting, and standing positions. In the present study, five methods were used which included: 1) supine lying, abduction of hand, and opening thighs, 2) supine lying, abduction of hands, and closing the angle of thighs, 3) sitting, abducting the hands, and closing the angle of thighs, 4) standing and bending the trunk forward, and 5) standing against the wall and opening the angle of thighs. The duration of each method varied between 5 and 15 minutes. These movements were performed for 6 weeks and three sessions per each week. Furthermore, in the present study, oral instructions and the manual booklet were used to keep the proper alignment and prevent compensatory movements of the patients. ${ }^{17}$

\section{Statistical Methods}

Normality and variance homogeneity of data were tested using the Shapiro-Wilk and Levene tests, respectively.

Table 1. Movement Control Test

\begin{tabular}{l}
\hline Waiters bow: Flexion of the hips in upright standing without movement of the low back. ${ }^{30}$ \\
$\begin{array}{l}\text { Pelvic tilt: Dorsal tilt of pelvis actively in upright standing. }{ }^{30} \\
\text { One leg stance: From normal standing to one leg stance: measurement of lateral movement of the belly button. (Position: feet one third of trochanter } \\
\text { distance apart). }{ }^{30}\end{array} \quad$ Sitting knee extension: Upright sitting with neutral lumbar lordosis; extension of the knee without movement (flexion) of the low back. ${ }^{30}$ \\
$5 \quad$ Quadruped position: Transfer of the pelvis backwards and forwards (rocking) keeping low back in neutral. Starting position $90^{\circ}$ hip flexion. ${ }^{30}$ \\
$6 \quad$ Prone lying active knee flexion. ${ }^{30}$
\end{tabular}




\begin{tabular}{|c|c|}
\hline Rectus Femoris & $\begin{array}{l}\text { The patient was placed on the bed in a supine position, such that the leg under study was out hanging off the bedside. The goniometer } \\
\text { center (Axis) was placed on the lateral epicondyle. Its stationary arm was aligned with greater trochanter. Its moving arm was placed } \\
\text { along the lateral malleolus. The patient's knee was flexed until he felt an extreme sense of stretching and pain in the anterior knee. At } \\
\text { this stage, the goniometer angle, which represented muscle flexibility, was read and recorded.32 }\end{array}$ \\
\hline $\begin{array}{l}\text { External rotator } \\
\text { muscles }\end{array}$ & $\begin{array}{l}\text { The patient lied on the stomach and flexed the knee up to } 90^{\circ} \text {. The goniometer center was placed on the tuberosity of the tibia. Its } \\
\text { stationary arm was along the vertical line. Its moving arm was placed parallel to the tibia. Next, while the femur was fixed using one } \\
\text { hand, the shin was put inwards by the other hand. At this stage, the angle between the vertical line and length of the fibula bone was } \\
\text { read, which represented flexibility of the external rotator muscles. }{ }^{33}\end{array}$ \\
\hline $\begin{array}{l}\text { Tensor fasciae } \\
\text { latae muscle }\end{array}$ & $\begin{array}{l}\text { The patient was placed in a supine position. The center of the goniometer was placed over the Anterior Superior Iliac Spine (ASIS) of } \\
\text { the extremity being measured. The stationary arm was aligned with an imaginary line extending from one ASIS to the other. The moving } \\
\text { arm was aligned with the anterior midline of the femur, using the midline of the patella for reference. Alteration of the goniometer angle } \\
\text { represented muscle flexibility. }{ }^{34}\end{array}$ \\
\hline $\begin{array}{l}\text { Hamstring } \\
\text { muscle }\end{array}$ & $\begin{array}{l}\text { The patient was in a supine position. His leg was lifted with a straight knee passively until he felt a sense of the stretching and pain } \\
\text { behind the knee. The goniometer center was placed on the lateral epicondyle. The stationary arm was aligned with greater trochanter, } \\
\text { while the moving arm was placed parallel to the lateral malleolus. The angle between these two goniometer arms suggested muscle } \\
\text { flexibility. }{ }^{32}\end{array}$ \\
\hline
\end{tabular}

Descriptive and inferential statistics were used for data analysis. To investigate intra-group differences, paired $t$ test was used in SPSS software version 21.0. A $P$ value of less than 0.05 was considered statistically significant.

\section{Results}

A total of 27 men with NSLBP participated in this study. They had a mean age of $31.21 \pm 2.5147$ years, height of $166.44 \pm 6.11 \mathrm{~cm}$, and weight of $64.21 \pm 5.25 \mathrm{~kg}$. After initial evaluations and initiation of treatment, 3 patients were excluded from the study due to missing 3 consecutive training sessions.

The results of paired $t$-test indicated a significant difference in the pre- and post-test pain score $(P=0.004)$ (Table 3).

The results of paired $t$ test indicated that there were not any statistically significant differences in the flexibility of rectus femoris, tensor fasciae latae, and external rotators hip muscles. On the other hand, a significant difference was noted in the flexibility of hamstring muscle (Table 4).

\section{Discussion}

The aim of this study was to investigate the effect of the GPR method on the pain and flexibility of some hip muscles in men with NSLBP suffering from back movement control deficit. The results suggested that these exercises have had a significant effect on the level of pain. Further, the GPR method affected the extent of flexibility

Table 3. Paired Sample $T$ test for Comparing Pain Intensity after Global Postural Reeducation in Individuals with Non-specific Low Back Pain and Movement Control Deficit

\begin{tabular}{lcccc}
\hline Variable & $\begin{array}{c}\text { Mean } \pm \text { SD } \\
\text { Pre-test }\end{array}$ & $\begin{array}{c}\text { Mean } \pm \text { SD } \\
\text { Post-test }\end{array}$ & $\begin{array}{c}\text { Percentage } \\
\text { Changes }\end{array}$ & $\boldsymbol{P}$ Value \\
\hline Back pain & $6.32 \pm 1.54$ & $4.24 \pm 1.04$ & 32.91 & $0.004^{*}$ \\
\hline
\end{tabular}

Significant difference $(P \leq 0.05)$. of some hip muscles (rectus femoris, tensor fasciae latae, and external rotators), though it was not statistically significant. On the other hand, GPR significantly affected the flexibility of the hamstring muscle.

One of the important factors studied in this study is the flexibility of hip muscles (rectus femoris, Tensor fasciae latae, external rotators, and hamstring). One of the muscles of interest in this study was hamstring muscle. It is connected to ischial tuberosity, and shortening of this muscle causes posterior pelvic tilt, diminished back curve, and thus straight back, which results in NSLBP. According to the findings obtained by Zahednezhad et al, the pain intensity level in people with NSLBP has a correlation with the flexibility of the hip muscles. ${ }^{9}$ On the other hand, shortened hamstring muscles alongside this weakness can result in posterior pelvic tilt and diminished spinal curvature. ${ }^{9}$ The studies have also shown that any change in the normal pattern of the movements of the spine and hip can increase the incidence of NSLBP due to the muscular attachments to them. ${ }^{9}$ The results obtained from this study indicated that the GPR method was effective in improving the flexibility of hamstring muscle. Accordingly, since shortened hamstring muscle has the greatest relationship with the pain intensity. ${ }^{9}$ It can be stated that the enhanced flexibility of this muscle possibly contributes to pain mitigation.

Furthermore, considering the shortened muscle chain which was observed in the subjects suffering from NSLBP, we applied the GPR method (global active stretching along with isometric contractions) to the subjects. This method prevents the activity of agonist muscles and stimulates antagonist muscles, causing the muscle spindle to stretch. The tonic muscles represent two-thirds of the total of our musculature, they are quite fibrous and of slow contraction and have a high tone. These characteristics reinforce their resistance and make it possible for part 
Table 4. Paired Sample $T$ test for Comparing Flexibility of Hip Muscles After Global Postural Reeducation in Individuals With Non-specific Low Back Pain and Movement Control Deficit

\begin{tabular}{lcccccccc}
\hline \multirow{2}{*}{ Muscle } & \multicolumn{2}{c}{ Mean \pm SD } & \multicolumn{2}{c}{ Mean \pm SD } & \multicolumn{2}{c}{ Percentage Changes (\%) } \\
\cline { 2 - 8 } & Right Leg & Left Leg & Right Leg & Left Leg & Right Leg & Left Leg & Right Leg & Left Leg \\
\hline Rectus femoris & $43.56 \pm 2.11$ & $41.34 \pm 3.22$ & $43.32 \pm 1.23$ & $40.3 \pm 2.54$ & 0.55 & 2.51 & 0.457 \\
Tensor fascia latae & $26.49 \pm 3.19$ & $25.39 \pm 3.41$ & $25.45 \pm 5.28$ & $26.04 \pm 3.32$ & 3.92 & -2.56 & 0.334 \\
Hamstring & $82.44 \pm 5.06$ & $85.30 \pm 7.11$ & $90.43 \pm 6.36$ & $91.35 \pm 5.38$ & -9.69 & -7.09 & $0.003 *$ & $0.003 *$ \\
External rotators & $37.42 \pm 6.43$ & $38.56 \pm 3.58$ & $36.31 \pm 6.83$ & $38.38 \pm 6.23$ & 2.96 & 0.46 & 0.217 & 0.328 \\
\hline
\end{tabular}

*Significant difference $(P \leq 0.05)$

of their fibers to remain in permanent contraction and in constant interaction with gravity. Its characteristics guarantee us the possibility of being erect and achieving stability, hence the reason why they are commonly known as postural muscles. ${ }^{35,36}$ This group tends to stiffen and shorten, and its injury is commonly the result of overuse or misuse. Additionally, due to their arrangement in the joints, when they become more hypertonic and shorten, they add a compression and shearing component to joints that could evolve in a morphological change and therefore an unbalance of tensions and possibly pain. The GPR method has been designed considering the physiology of each muscle group and focuses on the elongation of the tonic muscles conditioned at the same time by an eccentric contraction of the dynamic muscles while achieving decompression and correct alignment of the joint segments. ${ }^{35,36}$

In patients with NSLBP, this can result in improved mobility of the spine and reduced bodily pain. ${ }^{37}$ Among the possible reasons for the effectiveness of these exercises in improving the flexibility of hamstring muscles, one can mention that the reeducation exercises induce posterior and anterior muscle chain to stretch and affect the flexibility of hip muscles, which could possibly be effective in mitigating pain intensity. Although we cannot definitely say that GPR exercises alone are effective for patients with NSLBP, these exercises can be considered as an important part of the therapeutic method for patients with NSLBP.

In our research, the reason for the insignificant effect of GPR on the improvement of the flexibility of tensor fasciae, latae, rectus femoris, and external rotator muscles could be attributed to the short duration of the exercise program (6 weeks). On the other hand, GPR significantly improved pain and flexibility of hamstring muscles in patients with NSLBP. Evidently, this type of exercises prevents the activity of agonist muscles and stimulates antagonist muscles, causing the muscle spindle to stretch. In people with chronic NSLBP, this can result in improved mobility of the spine, flexibility, and diminished pain. The findings of our study on the improvement of pain were similar to those obtained by Bonetti et al, who evaluated the effects of GPR method on pain and functional disability. In this study, GPR method improved the pain and functional disability in the patients with NSLBP. ${ }^{17}$

\section{Conclusion}

Overall, the results of this study suggest the positive role of GPR method in improving pain in patients with NSLBP suffering from back movement control deficit. In addition, no dissatisfaction or side effect was reported by the patients in this study.

This finding alongside the results of previous studies can be considered a great contribution to exercise therapy in these patients. One of the limitations of this study was the absence of a control group.

\section{Ethical Approval}

This study was approved by the Ethics Committee of Sports Sciences Research Institute (Code: IR.SSRI. REC.1397.267).

\section{Conflict of Interest Disclosure}

The authors declare that they have no competing interests.

\section{Informed Consent}

All participants were informed about procedures, and they signed an informed consent form prior to participation in study procedures, in accordance with the declaration of Helsinki.

\section{References}

1. Mohseni Bandpei MA, Ehsani F, Behtash H, Ghanipour M. Occupational low back pain in primary and high school teachers: prevalence and associated factors. J Manipulative Physiol Ther. 2014;37(9):702-8. doi: 10.1016/j. jmpt.2014.09.006.

2. Jin K, Sorock GS, Courtney TK. Prevalence of low back pain in three occupational groups in Shanghai, People's Republic of China. J Safety Res. 2004;35(1):23-8. doi: 10.1016/j.jsr.2003.11.002.

3. Arab AM, Behbahani RB, Lorestani L, Azari A. Assessment of pelvic floor muscle function in women with and without low back pain using transabdominal ultrasound. Man Ther. 2010;15(3):235-9. doi: 10.1016/j.math.2009.12.005.

4. Chou R. Low back pain (chronic). BMJ Clin Evid. 2010;2010.

5. Mok NW, Yeung EW, Cho JC, Hui SC, Liu KC, Pang CH. 
Core muscle activity during suspension exercises. J Sci Med Sport. 2015;18(2):189-94. doi: 10.1016/j.jsams.2014.01.002.

6. Luomajoki HA, Bonet Beltran MB, Careddu S, Bauer CM. Effectiveness of movement control exercise on patients with non-specific low back pain and movement control impairment: a systematic review and meta-analysis. Musculoskelet Sci Pract. 2018;36:1-11. doi: 10.1016/j. msksp.2018.03.008.

7. du Rose A, Breen A, Breen A. Relationships between muscle electrical activity and the control of inter-vertebral motion during a forward bending task. J Electromyogr Kinesiol. 2018;43:48-54. doi: 10.1016/j.jelekin.2018.08.004.

8. Lomas-Vega R, Garrido-Jaut MV, Rus A, Del-PinoCasado R. Effectiveness of global postural re-education for treatment of spinal disorders: a meta-analysis. Am J Phys Med Rehabil. 2017;96(2):124-30. doi: 10.1097/ phm.0000000000000575.

9. Zahednezhad S, Salehi R, Tajali S, Borji A. Correlation between pain intensity and disability level with some of the impairments in patients with nonspecific low back pain. Journal of Ilam University of Medical Sciences. 2013;21(2):10-20. [Persian].

10. Hayden JA, van Tulder MW, Malmivaara A, Koes BW. Exercise therapy for treatment of non-specific low back pain. Cochrane Database Syst Rev. 2005(3):CD000335. doi: 10.1002/14651858.CD000335.pub2.

11. Stevenson JM, Weber CL, Smith JT, Dumas GA, Albert WJ. A longitudinal study of the development of low back pain in an industrial population. Spine (Phila Pa 1976). 2001;26(12):1370-7. doi: 10.1097/00007632-20010615000022 .

12. van der Velde G, Mierau D. The effect of exercise on percentile rank aerobic capacity, pain, and self-rated disability in patients with chronic low-back pain: a retrospective chart review. Arch Phys Med Rehabil. 2000;81(11):1457-63. doi: 10.1053/apmr.2000.9629.

13. Gordon R, Bloxham S. A systematic review of the effects of exercise and physical activity on non-specific chronic low back pain. Healthcare (Basel). 2016;4(2). doi: 10.3390/ healthcare4020022.

14. Leo Rathinaraj AS, Sreeja MT, Arun B, Sundar KS, Premlal R. A surface electromyographic study to assess the effect of spinal segmental stabilization [multifidus] exercise program in chronic mechanical low back pain patients. Eur Orthop Traumatol. 2012;3(3):161-8. doi: 10.1007/s12570012-0113-y.

15. Nezhad Roomezi S, Rahnama N, Habibi A, Negahban $H$. The effect of core stability training on pain and performance in women patients with non-specific chronic low back pain. Journal of Research in Rehabilitation Sciences. 2012;8(1):57-64. [Persian].

16. Souchard PE, Meli O, Sgamma D, Pillastrini P. Rieducazione posturale globale. EMC - Medicina Riabilitativa. 2009;16(3):1-10. doi: 10.1016/S1283-078X(09)70207-X.

17. Bonetti F, Curti S, Mattioli S, et al. Effectiveness of a 'Global Postural Reeducation' program for persistent low back pain: a non-randomized controlled trial. BMC Musculoskelet Disord. 2010;11:285. doi: 10.1186/1471-2474-11-285.

18. Haroon N, Inman RD. Ankylosing spondylitis - new criteria, new treatments. Bull NYU Hosp Jt Dis. 2010;68(3):171-4.
19. Stillman BC. Making sense of proprioception: the meaning of proprioception, kinaesthesia and related terms. Physiotherapy. 2002;88(11):667-76. doi: 10.1016/S00319406(05)60109-5.

20. Loudon J. Therapeutic exercise: treatment planning for progression. Phys Ther. 2007;87(5):616-7. doi: 10.2522/ ptj.2007.87.5.616.

21. O'Sullivan P. Diagnosis and classification of chronic low back pain disorders: maladaptive movement and motor control impairments as underlying mechanism. Man Ther. 2005;10(4):242-55. doi: 10.1016/j.math.2005.07.001.

22. Hill JC, Dunn KM, Lewis M, et al. A primary care back pain screening tool: identifying patient subgroups for initial treatment. Arthritis Rheum. 2008;59(5):632-41. doi: 10.1002/art.23563.

23. Barrett E, McCreesh K, Lewis J. Reliability and validity of non-radiographic methods of thoracic kyphosis measurement: a systematic review. Man Ther. 2014;19(1):10-7. doi: 10.1016/j.math.2013.09.003.

24. Coelho DM, Bonagamba GH, Oliveira AS. Scoliometer measurements of patients with idiopathic scoliosis. Braz J Phys Ther. 2013;17(2):179-84. doi: 10.1590/s141335552012005000081.

25. Faul F, Erdfelder E, Lang AG, Buchner A. G*Power 3: a flexible statistical power analysis program for the social, behavioral, and biomedical sciences. Behav Res Methods. 2007;39(2):175-91. doi: 10.3758/bf03193146.

26. Faul F, Erdfelder E, Buchner A, Lang AG. Statistical power analyses using $G^{\star}$ Power 3.1: tests for correlation and regression analyses. Behav Res Methods. 2009;41(4):114960. doi: 10.3758/brm.41.4.1149.

27. Luomajoki H, Kool J, de Bruin ED, Airaksinen O. Improvement in low back movement control, decreased pain and disability, resulting from specific exercise intervention. Sports Med Arthrosc Rehabil Ther Technol. 2010;2:11. doi: 10.1186/1758-2555-2-11.

28. Kopec JA, Esdaile JM, Abrahamowicz M, et al. The Quebec Back Pain Disability Scale. Measurement properties. Spine (Phila Pa 1976). 1995;20(3):341-52. doi: 10.1097/00007632199502000-00016.

29. Oesch P, Kool J, Hagen KB, Bachmann S. Effectiveness of exercise on work disability in patients with non-acute non-specific low back pain: systematic review and metaanalysis of randomised controlled trials. J Rehabil Med. 2010;42(3):193-205. doi: 10.2340/16501977-0524.

30. Luomajoki H, Kool J, de Bruin ED, Airaksinen O. Movement control tests of the low back; evaluation of the difference between patients with low back pain and healthy controls. BMC Musculoskelet Disord. 2008;9:170. doi: 10.1186/1471-2474-9-170.

31. Wewers ME, Lowe NK. A critical review of visual analogue scales in the measurement of clinical phenomena. Res Nurs Health. 1990;13(4):227-36. doi: 10.1002/nur.4770130405.

32. Reese NB, Bandy WD. Joint Range of Motion and Muscle Length Testing. Philadelphia: Saunders; 2002.

33. Sahrmann S. Diagnosis and Treatment of Movement Impairment Syndromes. Mosby; 2002.

34. Norkin CC, White DJ. Measurement of Joint Motion: A Guide to Goniometry. Philadelphia: F.A. Davis Company; 2016. 
35. Mercado D. Assessment of the Effects of Global Postural ReEducation on Musicians with Nonspecific Musculoskeletal Pain as Assessed by Questionnaires and Infrared Thermography [dissertation]. University of Ottawa; 2018.

36. Page P, Frank CC, Lardner R. Assessment and Treatment of Muscle Imbalance: The Janda Approach. Windsor, ON:
Human Kinetics; 2009.

37. Silva EM, Andrade SC, Vilar MJ. Evaluation of the effects of Global Postural Reeducation in patients with ankylosing spondylitis. Rheumatol Int. 2012;32(7):2155-63. doi: 10.1007/s00296-011-1938-3. 